\title{
Exceptional Groups and Their Modular Forms
}

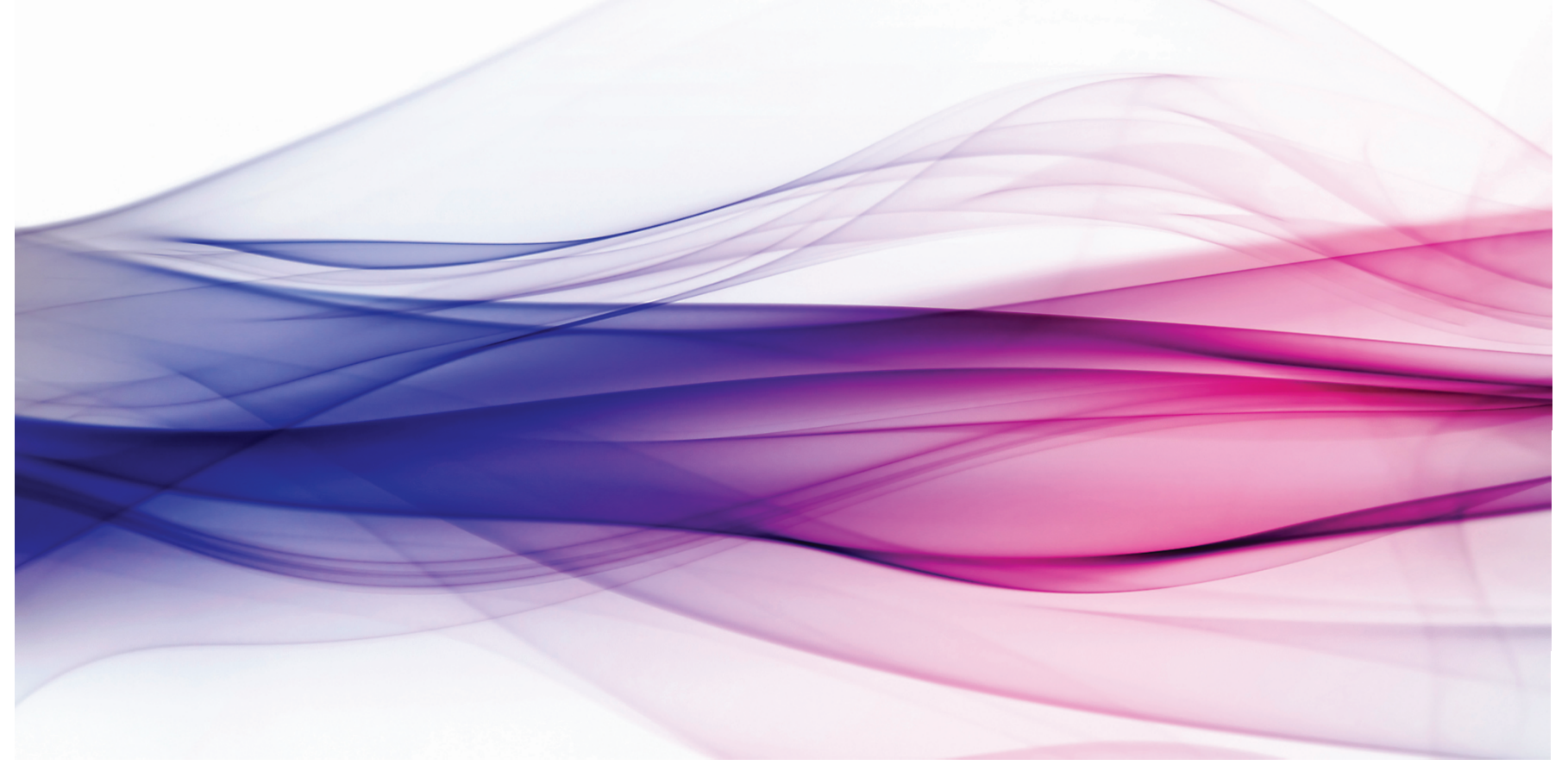

\section{Aaron Pollack}

\section{Introduction}

It is an old theorem of Lagrange that every nonnegative integer can be expressed as the sum of four squares of integers. That is, if $n \in \mathbf{Z}_{\geq 0}$, then there exist integers $x_{1}, x_{2}, x_{3}, x_{4}$ so that $n=x_{1}^{2}+x_{2}^{2}+x_{3}^{2}+x_{4}^{2}$. For the sake of comparison, note that 7 is not the sum of three squares, so not every integer can be expressed as $x_{1}^{2}+x_{2}^{2}+x_{3}^{2}$.

If $n \in \mathbf{Z}_{\geq 0}$ and $m>0$ is a positive integer, denote by $r_{m}(n)$ the number of ways one can express $n$ as the sum of $m$ squares of integers. That is, define $r_{m}(n)$ to be the size of the set

$$
\left\{\left(x_{1}, x_{2}, \ldots, x_{m}\right) \in \mathbf{Z}^{m}: x_{1}^{2}+x_{2}^{2}+\cdots+x_{m}^{2}=n\right\} .
$$

Aaron Pollack is an assistant professor at the University of California, San Diego. His email address is apo11ack@ucsd.edu.

The author was supported by the Simons Foundation via Collaboration Grant number 585147.

Communicated by Notices Associate Editor Steven Sam.

For permission to reprint this article, please contact:

reprint-permission@ams.org.

DOI: https://doi.org/10.1090/noti2226
Then Lagrange's theorem says that $r_{4}(n) \geq 1$ for every positive integer $n$. In fact, there is a beautiful formula due to Jacobi for $r_{4}(n)$. Denote by $\sigma(n)=\sum_{d \mid n} d$ the sum of the divisors of the integer $n$. Then $r_{4}(n)=8 \sigma(n)$ if $n$ is odd and $r_{4}(n)=24 \sigma(n)$ if $n$ is even.

Where does such a nice formula come from? The contemporary explanation is that this formula comes from the theory of modular forms. Consider the power series $\sum_{n \geq 0} r_{4}(n) q^{n} \in \mathbf{Z}[[q]]$ in the variable $q$. If $z$ is in the complex upper half-plane $\mathfrak{h}=\{x+i y: x, y \in \mathbf{R}, y>0\}$ and $q=e^{2 \pi i z}$, then $\theta_{4}(z):=\sum_{n \geq 0} r_{4}(n) q^{n}$ becomes a holomorphic function on $\mathfrak{h}$. The function $\theta_{4}(z)$ is an example of a modular form (defined below), which amounts to the fact that $\theta_{4}(z+1)=\theta_{4}(z)$ and $\theta_{4}\left(-\frac{1}{4 z}\right)=(4 z)^{2} \theta_{4}(z)$ as functions on $\mathfrak{h}$. The first functional equation is obvious but the second is not. These symmetries go a long way to proving the relationship between $r_{4}(n)$ and the divisor function $\sigma(n)$.

Note that $z \mapsto z+1$ and $z \mapsto-\frac{1}{4 z}$ are the linear fractional transformations of $\mathfrak{h}$ associated to the matrices $\left(\begin{array}{ll}1 & 1 \\ 0 & 1\end{array}\right)$ 
and $\left(\begin{array}{cc}0 & -1 \\ 4 & 0\end{array}\right)$. There is a finite index subgroup $\Gamma$ of $\mathrm{SL}_{2}(\mathbf{Z})$ so that $\theta_{4}(z)$ satisfies a functional equation associated to every element $\gamma \in \Gamma$. Namely, if $\gamma=\left(\begin{array}{ll}a & b \\ c & d\end{array}\right) \in \Gamma$, then $\theta_{4}\left(\frac{a z+b}{c z+d}\right)=(c z+d)^{2} \theta_{4}(z)$. In this way, one can think of $\theta_{4}(z)$ as a special function associated to the group $\mathrm{SL}_{2}$. The reader can see [Zag08] and [Ser73] for an introduction to modular forms, and in particular [Zag08, Section 3] for a proof of Jacobi's formula via $\theta_{4}(z)$.

The modular form $\theta_{4}(z)$ is a special example of what is called an automorphic form. Automorphic forms are functions that have large groups of discrete symmetries and satisfy particular types of differential equations. (The function $\theta_{4}(z)$ satisfies the Cauchy-Riemann equations.) This article is about automorphic forms, with a special emphasis on those automorphic forms whose groups of symmetries are connected to the exceptional Lie groups. We do not suppose the reader knows anything about modular forms or exceptional groups, only the representation theory of compact groups and a little algebraic geometry.

\section{Modular Forms}

We now dig into the definition of modular forms. Suppose that $k>0$ is a positive integer and $\Gamma \subseteq \mathrm{SL}_{2}(\mathbf{Z})$ is a finite index subgroup. We assume that there exists a positive integer $N$ so that $\Gamma$ contains the subgroup $\Gamma(N)$ of $\mathrm{SL}_{2}(\mathbf{Z})$ consisting of matrices congruent to 1 modulo $N$, i.e., $\Gamma(N)=\left\{\left(\begin{array}{ll}a & b \\ c & d\end{array}\right) \in \mathrm{SL}_{2}(\mathbf{Z}): a, d \equiv 1(\bmod N), b, c \equiv 0\right.$ $(\bmod N)\}$. A modular form of weight $k$ for $\Gamma$ is a holomorphic function $f: \mathfrak{h} \rightarrow \mathbf{C}$ that is semi-invariant under $\Gamma$ and doesn't grow too quickly. More precisely, a holomorphic function $f: \mathfrak{h} \rightarrow \mathbf{C}$ is a modular form of weight $k$ for $\Gamma$ if

1. $f(\gamma z)=(c z+d)^{k} f(z)$ for all $\gamma=\left(\begin{array}{ll}a & b \\ c & d\end{array}\right) \in \Gamma$,

2. the function $y^{k / 2}|f(z)|$ grows at most polynomially with $y$ on $\Gamma \backslash \mathfrak{h}$. (Note that $y^{k / 2}|f(z)|$ is $\Gamma$-invariant, as $\operatorname{Im}(\gamma z)=\operatorname{Im}(z)|c z+d|^{-2}$.)

We denote by $M_{k}(\Gamma)$ the space of modular forms of weight $k$ for $\Gamma$. For $k$ and $\Gamma$ fixed, the space $M_{k}(\Gamma)$ is a finitedimensional (sometimes 0 ) complex vector space.

Note that if $f: \mathfrak{h} \rightarrow \mathbf{C}$ is a holomorphic function, then $f(z) d z$ is $\Gamma$-invariant if and only if $f(\gamma z)=(c z+d)^{2} f(z)$, so weight- 2 modular forms are in bijection with a subspace of the holomorphic differential-one forms on $Y_{\Gamma}:=\Gamma \backslash \mathfrak{h}$.

One of the first things that must be said about modular forms is that they have a Fourier expansion: suppose for simplicity that $\Gamma=\mathrm{SL}_{2}(\mathbf{Z})$. Then the elements $\left(\begin{array}{ll}1 & n \\ 0 & 1\end{array}\right) \in \Gamma$ for $n \in \mathbf{Z}$ and thus the condition $f(\gamma z)=(c z+d)^{k} f(z)$ becomes $f(z+n)=f(z)$. As $f$ is holomorphic, this implies that $f(z)=\sum_{n \in \mathbf{Z}} a_{f}(n) e^{2 \pi i n z}$ for complex numbers $a_{f}(n) \in \mathbf{C}$. As the reader can immediately check, condition 2 above implies that $a_{f}(n)=0$ if $n$ is negative. That is, $f(z)=\sum_{n \geq 0} a_{f}(n) e^{2 \pi i n z}$.
2.1. Examples. The special function $\theta_{4}(z)$ described in the introduction is a modular form of weight 2 . More generally, if $m \geq 0$ is a nonnegative integer, then one can define $\theta_{m}(z)=\sum_{n \geq 0} r_{m}(n) q^{n}$. When $m$ is even, $\theta_{m}(z)$ is a modular form of weight $m / 2$.

A simpler set of examples of modular forms are the socalled Eisenstein series $E_{k}(z)$. Suppose $\Gamma \subseteq \mathrm{SL}_{2}(\mathbf{Z})$ is fixed, and denote $\Gamma_{\infty}=\left\{\left(\begin{array}{ll}1 & n \\ 0 & 1\end{array}\right) \in \Gamma\right\}$. If $k \geq 4$, one defines

$$
E_{k}(z ; \Gamma)=\sum_{\gamma \in \Gamma_{\infty} \backslash \Gamma}(c z+d)^{-k} .
$$

In the sum above, $\gamma=\left(\begin{array}{ll}a & b \\ c & d\end{array}\right)$ and the condition $k \geq 4$ ensures that the sum converges absolutely to a holomorphic function on $\mathfrak{h}$. Note that if $\gamma_{\infty} \in \Gamma_{\infty}$, then $\gamma_{\infty} \gamma=\left(\begin{array}{c}* \\ c \\ c\end{array}\right)$, so that the sum (1) is well-defined.

These functions have simple Fourier expansions. For example, if $\Gamma=\mathrm{SL}_{2}(\mathbf{Z})$, then $E_{k}(z):=E_{k}(z ; \Gamma)$ has Fourier expansion

$$
E_{k}(z)=1+\alpha_{k} \sum_{n \geq 1}\left(\sum_{d \mid n} d^{k-1}\right) q^{n}
$$

for a nonzero rational number $\alpha_{k}$. By relating $\theta_{4}(z)$ with a certain weight-2 Eisenstein series $E_{2}(z ; \Gamma)$, one can prove Jacobi's formula for $r_{4}(n)$.

The examples above all have the feature that the constant term $a_{f}(0) \neq 0$, so that the function $y^{k / 2}|f(z)|$ grows as $y \rightarrow \infty$. The subspace of modular forms that decay as $y \rightarrow \infty$ occupies a special place in the theory. More precisely, the noncompact complex curve $Y_{\Gamma}$ can be compactified to a curve $X_{\Gamma}$. The set $X_{\Gamma} \backslash Y_{\Gamma}$ is finite, and is called the cusps of the modular curve $X_{\Gamma}$. The modular forms $f$ of weight $k$ for which the function $y^{k / 2}|f(z)|$ on $Y_{\Gamma}$ decays towards the cusps are called cusp forms, denoted by $S_{k}(\Gamma) \subseteq M_{k}(\Gamma)$. Ramanujan defined the following beautiful cusp form of weight $k=12$ :

$$
\Delta(z):=q \prod_{n \geq 1}\left(1-q^{n}\right)^{24}=\sum_{n \geq 1} \tau(n) q^{n} .
$$

The numbers $\tau(n)$ are by definition the complex numbers that make this an equality. It is not obvious that $\Delta(z)$ is a modular form. Another fact that is not clear from the definition is that $\tau(m n)=\tau(m) \tau(n)$ if the positive integers $m$ and $n$ are relatively prime. To give the reader a sense for a simple statement which is already not known, we remark that it is conjectured that $\tau(n) \neq 0$ for all $n$.

2.2. The group $\mathrm{SL}_{2}$. As suggested in the introduction, modular forms should be thought of as functions associated to the group $\mathrm{SL}_{2}$. To make this precise, recall that the action of $\mathrm{SL}_{2}(\mathbf{R})$ on $\mathfrak{h}$ via linear fractional transformations is transitive, and that the stabilizer in $\mathrm{SL}_{2}(\mathbf{R})$ of the point $\sqrt{-1} \in \mathfrak{h}$ is the special orthogonal group of size two:

$$
\mathrm{SO}(2)=\left\{k_{\theta}:=\left(\begin{array}{cc}
\cos (\theta) & \sin (\theta) \\
-\sin (\theta) & \cos (\theta)
\end{array}\right): \theta \in \mathbf{R}\right\} .
$$


That is, one has an $\mathrm{SL}_{2}(\mathbf{R})$-equivariant identification $\mathfrak{h} \simeq$ $\mathrm{SL}_{2}(\mathbf{R}) / \mathrm{SO}(2)$.

For $g=\left(\begin{array}{ll}a & b \\ c & d\end{array}\right) \in \mathrm{SL}_{2}(\mathbf{R})$ and $z \in \mathfrak{h}$, set $j(g, z)=$ $c z+d$. The reader can easily check that for $g_{1}, g_{2} \in \mathrm{SL}_{2}(\mathbf{R})$, $j\left(g_{1} g_{2}, z\right)=j\left(g_{1}, g_{2} z\right) j\left(g_{2}, z\right)$. Now if $f \in M_{k}(\Gamma)$ is a weight $k$ modular form, then one can define a closely related function $\phi_{f}: \Gamma \backslash \mathrm{SL}_{2}(\mathbf{R}) \rightarrow$ C. Namely, set

$$
\phi_{f}(g)=j(g, i)^{-k} f(g \cdot i) .
$$

Then if $\gamma \in \Gamma$ and $z=g \cdot i$,

$$
\begin{aligned}
\phi_{f}(\gamma g) & =j(\gamma g, i)^{-k} f(\gamma \cdot z) \\
& =j(\gamma, z)^{-k} j(g, i)^{-k} j(\gamma, z)^{k} f(z)=\phi_{f}(g)
\end{aligned}
$$

so that $\phi_{f}$ is left- $\Gamma$-invariant. Similarly, one can easily verify that if $k_{\theta} \in \mathrm{SO}(2)$ as in (2), then $\phi_{f}\left(g k_{\theta}\right)=e^{i k \theta} \phi_{f}(g)$ for all $g \in \mathrm{SL}_{2}(\mathbf{R})$.

To summarize, set $G=\mathrm{SL}_{2}(\mathbf{R})$ and fix $\Gamma \subseteq G$ and $k$ an integer. Then one can consider the space $M_{k, \infty}(\Gamma)$ of smooth functions $\varphi: G \rightarrow \mathbf{C}$ defined as follows.

Definition 1. A smooth function $\varphi: G \rightarrow \mathbf{C}$ is in $M_{k, \infty}(\Gamma)$ if

1. $\varphi(\gamma g)=\varphi(g)$ for all $g \in G$ and $\gamma \in \Gamma$,

2. $\varphi\left(g k_{\theta}\right)=e^{i k \theta} \varphi(g)$ for all $g \in G$ and $k_{\theta} \in \mathrm{SO}(2)$,

3. $\varphi \in C^{\infty}(\Gamma \backslash G)$ has "moderate growth" (condition 2 above) in an appropriate sense.

We have just defined an injection $M_{k}(\Gamma) \hookrightarrow M_{k, \infty}(\Gamma)$. The space $M_{k}(\Gamma)$ can then be picked out inside $M_{k, \infty}(\Gamma)$ by finding the functions that (essentially) satisfy the CauchyRiemann equations.

Put in this context, one can immediately generalize: what are the elements of $M_{k, \infty}(\Gamma)$ (for some $k$ ) that do not come from holomorphic functions on $\mathfrak{h}$ ? How about analogously defined spaces for groups other than $\mathrm{SL}_{2}(\mathbf{R})$ ? Both of these generalizations are important. The relevant space of functions are called "automorphic forms." We will concentrate our exposition on the latter question of generalizing from $\mathrm{SL}_{2}$ to other groups.

\section{Automorphic Forms}

So that the reader can get some sense of the broader picture, let us briefly say a bit about automorphic forms more generally. As mentioned above, one arrives at the notion of automorphic forms by appropriately replacing the pair $\left(\mathrm{SL}_{2}(\mathbf{R}), \Gamma\right)$ by other pairs $\left(G, \Gamma_{G}\right)$ in the definition of $M_{k, \infty}(\Gamma)$ above, where $G$ is a reductive Lie group (reviewed momentarily) and $\Gamma$ is an appropriate discrete subgroup of $G$.

3.1. Reductive groups. At first pass, reductive groups are perhaps best understood by example, as opposed to by definition. Set $I_{p, q}=\left(\begin{array}{ll}1_{p} & \\ & -1_{q}\end{array}\right)$ and $J_{n}=\left(\begin{array}{cc}{ }_{-1} 1_{n} \\ -{ }_{n}\end{array}\right)$. Some examples of reductive Lie groups $G$ are:
- $\mathrm{GL}_{n}(\mathbf{R})$,

- $\mathrm{SL}_{n}(\mathbf{R})$,

- $\mathrm{SO}(p, q)=\left\{g \in \mathrm{SL}_{p+q}(\mathbf{R}): g I_{p, q} g^{t}=I_{p, q}\right\}$, the special orthogonal group of a vector space with a non-degenerate quadratic form of signature $(p, q)$,

- $U(p, q)=\left\{g \in \mathrm{GL}_{p+q}(\mathbf{C}): g I_{p, q} \bar{g}^{t}=I_{p, q}\right\}$, the similarly defined unitary group, and

- $\operatorname{Sp}_{2 n}(\mathbf{R})=\left\{g \in \mathrm{SL}_{2 n}(\mathbf{R}): g J_{n} g^{t}=J_{n}\right\}$, the group of automorphisms of a $2 n$-dimensional real vector space preserving a nondegenerate alternating bilinear form.

It is good to compare and contrast reductive groups with compact Lie groups. Recall that the compact finitedimensional Lie groups are classified, and many come in infinite families such as the special orthogonal group $\mathrm{SO}(n)$ and the unitary group $U(n)$. If $K$ is a compact Lie group, then

- any finite-dimensional representation of $K$ can be written as a finite sum of irreducible representations, and

- every irreducible (unitary) representation of $K$ is finite dimensional.

A reductive Lie group is a group $G$ that possesses this first property, but almost never possesses the second: if $G \neq\{1\}$ is a noncompact reductive Lie group, there are no faithful finite-dimensional irreducible unitary representations ${ }^{1}$ of $G$. All compact Lie groups are reductive, but automorphic forms are generally only interesting to consider for noncompact groups $G$. The reader should keep in mind $G=\mathrm{SL}_{n}(\mathbf{R})$ and $G=\mathrm{SO}(p, q)$ with $p q \neq 0$ as examples of reductive groups.

3.2. Spaces of functions. For reductive groups $G$, most of the discrete subgroups $\Gamma_{G}$ that arise in the theory of automorphic forms are what are known as arithmetic subgroups, and they have the property that $\Gamma_{G} \backslash G$ is sometimes (but not usually) compact, but always has finite $G$ invariant volume. Without giving a precise definition, one should think of $\Gamma_{G}$ as being defined in the same way as $G$ except with the real numbers $\mathbf{R}$ replaced by the integers Z. For example, if $G=\mathrm{SL}_{n}(\mathbf{R})$, then $\Gamma_{G}$ could be $\mathrm{SL}_{n}(\mathbf{Z})$ $\left(=\mathrm{SL}_{n}(\mathbf{R}) \cap M_{n}(\mathbf{Z})\right)$ or one of its finite index subgroups.

With $G$ and $\Gamma_{G}$ fixed, one can consider various spaces of functions on the manifold $\Gamma_{G} \backslash G$, for example $L^{2, \infty}\left(\Gamma_{G} \backslash G\right)$, the space of smooth, complex-valued $L^{2}$-functions on $\Gamma_{G} \backslash G$. One also considers the space $L^{m g, \infty}\left(\Gamma_{G} \backslash G\right)$, the smooth, moderate-growth functions on $G$. The group $G$ acts on $L^{2, \infty}\left(\Gamma_{G} \backslash G\right)$ via right translation: $(g \cdot \varphi)(x)=\varphi(x g)$, and this action affords an infinite-dimensional unitary representation of $G$.

\footnotetext{
${ }^{1}$ If $G=G_{1} \times G_{2}$ with $G_{1}$ compact, then $G$ has nonfaithful irreducible unitary representations via projection onto $G_{1}$.
} 
The automorphic forms $\mathcal{A}\left(G ; \Gamma_{G}\right)$ are, by definition, a certain dense, nice subspace of $L^{m g, \infty}\left(\Gamma_{G} \backslash G\right)$. To pick out this subspace, one imposes two extra conditions on functions $\varphi \in L^{m g, \infty}\left(\Gamma_{G} \backslash G\right)$. One condition is the analogue of condition 2 in Definition 1 above, and the other says that $\varphi$ satisfies sufficiently many differential equations that come from the group theory of $G$.

For the sake of completeness, we now spell out these conditions, although the reader might wish to skip to section 4. To make explicit the first condition, recall that every reductive Lie group $G$ has a maximal ${ }^{2}$ compact subgroup $K$, and any two maximal compact subgroups of $G$ are conjugate. Fixing one such $K$, we can restrict the $G$-action to $K$ and find the functions $\varphi \in L^{m g, \infty}\left(\Gamma_{G} \backslash G\right)$ whose translates by $K$ make up a finite-dimensional vector space. These functions are called $K$-finite. This is the analogue of condition 2 in Definition 1 above.

The second condition involves the universal enveloping algebra $\mathcal{U}\left(\mathfrak{g}_{\mathbf{C}}\right)$ of the complexified Lie algebra $\mathfrak{g}_{\mathbf{C}}$ of $G$. This infinite-dimensional noncommutative $\mathbf{C}$-algebra acts on $C^{\infty}\left(\Gamma_{G} \backslash G\right)$ by differentiating the right-translation action of $G$. The center $\boldsymbol{Z}\left(\mathfrak{g}_{\mathbf{C}}\right)$ of $\mathcal{U}\left(\mathfrak{g}_{\mathbf{C}}\right)$ is a commutative $\mathbf{C}$-algebra of finite type. For example, if $\mathfrak{g}=\mathfrak{g l}_{n^{\prime}}$ then

$$
z\left(\mathfrak{g}_{\mathbf{C}}\right) \simeq \mathbf{C}\left[t_{1}, \ldots, t_{n}\right]^{S_{n}},
$$

the symmetric polynomials in the variables $t_{1}, t_{2}, \ldots, t_{n}$. One can further consider functions $\varphi$ that are annihilated by an ideal of $z\left(\mathfrak{g}_{\mathrm{c}}\right)$ of finite codimension. Or in other words, for which $z\left(\mathfrak{g}_{\mathrm{C}}\right) \cdot \varphi$ is finite dimensional. Such functions are said to be $z\left(\mathfrak{g}_{C}\right)$-finite.

Putting everything together, set $\mathcal{A}\left(G ; \Gamma_{G}\right)$ as the space of smooth, moderate growth functions $\varphi: \Gamma_{G} \backslash G \rightarrow \mathbf{C}$ that are $K$-finite and $z\left(\mathfrak{g}_{\mathbf{C}}\right)$-finite. These are the $\Gamma_{G}$-invariant automorphic forms on $G$.

\section{L-Functions}

One of the most important aspects of automorphic forms is that they connect apparently disparate areas of mathematics. One way of making this connection precise is to use $L$-functions.

The reader is probably familiar with the Riemann zeta function $\zeta(s)$. Recall that if $s \in \mathbf{C}$ has $\operatorname{Re}(s)>1$, then one defines

$$
\zeta(s)=\sum_{n \geq 1} n^{-s}=\prod_{p}\left(1-p^{-s}\right)^{-1},
$$

where the latter product is over the prime numbers $p . L$ functions are generalizations of the Riemann zeta function. It is a famous fact that $\zeta(s)$ has a meromorphic continuation to the complex plane with a simple pole at $s=1$, and satisfies a functional equation relating $s$ to $1-s$. More specifically, define $\xi(s)=\pi^{-s / 2} \Gamma(s / 2) \zeta(s)$; then $\xi(s)$ has

\footnotetext{
${ }^{2}$ I.e., a compact subgroup that is not strictly included in any other compact subgroup.
}

simple poles at $s=1$ and $s=0$, is analytic elsewhere in the complex plane, and satisfies the exact functional equation $\xi(s)=\xi(1-s)$.

More generally, if $\left\{a_{n}\right\}_{n \geq 1}$ is a sequence of complex numbers, and if the sum $\sum_{n \geq 1} a_{n} n^{-s}$ converges absolutely for $\operatorname{Re}(s)>s_{0}$, one can then consider this function of $s$; it is called a Dirichlet series. An $L$-function is a very special type of Dirichlet series, and they are assigned to all sorts of "number-theoretic data," including modular forms and automorphic forms. Besides being representable as a sum $\sum_{n \geq 1} a_{n} n^{-s}, L$-functions share two other properties with the Riemann zeta function:

1. They can be representable as a product over primes $p$, called an "Euler product."

2. They satisfy a functional equation relating $s$ to $k-s$ for some real number $k$.

The Euler product comes from a multiplicativity property of the numbers $a_{n}$. Specifically, suppose that $a_{1}=1$ and $a_{n m}=a_{n} a_{m}$ when $n$ and $m$ are relatively prime. Then, in the range of absolute convergence, one has

$$
\begin{aligned}
\sum_{n \geq 1} a_{n} n^{-s} & =\prod_{p}\left(1+a_{p} p^{-s}+a_{p^{2}} p^{-2 s}+\cdots\right) \\
& =\prod_{p}\left(\sum_{k \geq 0} a_{p^{k}} p^{-k s}\right) .
\end{aligned}
$$

4.1. Automorphic $L$-functions. Denote by $\chi_{4}$ the unique nontrivial character on $(\mathbf{Z} / 4 \mathbf{Z})^{\times}$, i.e., $\chi_{4}(n)=1$ if $n \equiv 1$ $(\bmod 4)$ and $\chi_{4}(n)=-1$ if $n \equiv 3(\bmod 4)$. The simplest example of an $L$-function, beyond the Riemann zeta function, is

$$
\begin{aligned}
L\left(\chi_{4}, s\right) & :=1-3^{-s}+5^{-s}-7^{-s}+9^{-s}+\cdots \\
& =\prod_{p \equiv 1(4)}\left(1-p^{-s}\right)^{-1} \prod_{p \equiv 3(4)}\left(1+p^{-s}\right)^{-1} .
\end{aligned}
$$

More generally, suppose $N \geq 1$ is a positive integer and $\chi:(\mathbf{Z} / N \mathbf{Z})^{\times} \rightarrow \mathbf{C}^{\times}$is a character, i.e., $\chi\left(m_{1} m_{2}\right)=$ $\chi\left(m_{1}\right) \chi\left(m_{2}\right)$ for integers $m_{1}, m_{2}$ prime to $N$. Then one can extend $\chi$ to a function $\mathbf{Z} \rightarrow \mathbf{C}$ as $\chi(m)=0$ if $m$ and $N$ share a nontrivial common factor, and define

$$
L(\chi, s)=\sum_{n \geq 1} \chi(n) n^{-s}=\prod_{(p, N)=1}\left(1-\chi(p) p^{-s}\right)^{-1} .
$$

When $\chi=\chi_{4}$ one obtains the $L$-function (3).

It is an important fact that these $L$-functions have meromorphic (analytic) continuation and functional equation. In fact, Dirichlet [Dir37] used the properties of these $L$ functions to prove that if $a$ and $N$ are relatively prime positive integers, then there are infinitely many prime numbers congruent to $a$ modulo $N$.

The above examples have the property that the coefficients $a_{n}$ are completely multiplicative, i.e., $a_{m n}=a_{m} a_{n}$ for all $m, n$, not just when $m$ and $n$ are relatively prime. A more complicated example comes from the modular 
forms. Suppose $f(z)=\sum_{n \geq 1} a_{n} q^{n}$ is a modular form of weight $k$. For example, $f(z)$ could be Ramanujan's function $\Delta(z)$. The space of cusp forms $S_{k}\left(\mathrm{SL}_{2}(\mathbf{Z})\right)$ has a preferred basis, called the basis of Hecke eigenforms, and if $f(z)$ is such an eigenform, then one can associate an $L$ function to $f$. Specifically, suppose that $f(z)$ is normalized so that $a_{1}=1$. Then one defines $L(f, s)=\sum_{n \geq 1} a_{n} n^{-s}$. Setting $\Lambda(f, s)=\pi^{-s} \Gamma(s) L(f, s)$, then this function satisfies the functional equation $\Lambda(f, s)=\Lambda(f, k-s)$. In the special case of modular forms $f$ as above, the Euler product expansion of $L(f, s)$ looks as follows:

$$
L(f, s)=\prod_{p}\left(1-a_{p} p^{-s}+p^{k-1} p^{-2 s}\right)^{-1} .
$$

The above $L$-function is defined as a product over all the prime numbers $p$. It is technically very convenient in the theory of automorphic forms to also consider products as above over all by finitely many primes, whereby obtaining what are called partial $L$-functions. Throughout the rest of the text we blur the distinction between these different scenarios: some of the statements below are only correct when $L$-functions are replaced by partial $L$-functions.

4.2. Motivic $L$-functions. One can associate $L$-functions not only to modular forms, but more generally, to automorphic forms $\varphi$. However, one of the most basic ways to construct $L$-functions is via Galois theory. Suppose $K$ is a number field, with $K / \mathbf{Q}$ a Galois extension, and $\rho: \operatorname{Gal}(K / \mathbf{Q}) \rightarrow \mathrm{GL}_{n}(\mathbf{C})$ is a representation. One can construct an $L$-function $L(\rho, s)$ associated to this data as follows:

$$
L(\rho, s)=\prod_{p} \operatorname{det}\left(1-\rho\left(\text { Frob }_{p}\right) p^{-s}\right)^{-1} .
$$

Here $\mathrm{Frob}_{p}$ is a certain conjugacy class of $\operatorname{Gal}(\mathrm{K} / \mathbf{Q})$ associated to the prime $p$ of $\mathbf{Z}$, and note that the factor $\operatorname{det}\left(1-\rho\left(F_{r o b}\right) p^{-s}\right)$ is well-defined because the determinant makes the choice of representative in the conjugacy class irrelevant.

These $L$-functions $L(\rho, s)$ are known to have meromorphic continuation in $S$, as a consequence of results of Dirichlet and Brauer. Their holomorphy in $s$ is wide open.

Conjecture 2 (The Artin conjecture). Suppose $K / \mathbf{Q}$ is a $\mathrm{G} a$ lois extension and $\rho: \operatorname{Gal}(K / \mathbf{Q}) \rightarrow \mathrm{GL}_{n}(\mathbf{C})$ is an irreducible, nontrivial representation. Then $L(\rho, s)$ is an entire holomorphic function of $s$.

Algebro-geometric objects also have associated $L$ functions. More precisely, one can associate $L$-functions to the étale cohomology of smooth projective varieties over a number field. The first important example is that of an elliptic curve $E$ over $\mathbf{Q}$. For all but finitely many primes $p$, one can reduce $E$ modulo $p$ to obtain a curve over the finite field $\mathbf{F}_{p}$. Counting points modulo $p$, define the integer $a_{p}(E)$ as $a_{p}(E)=p+1-\# E\left(\mathbf{F}_{p}\right){ }^{3}$ One can package the integers $a_{p}(E)$ together as

$$
L(E, s)=\prod_{p}\left(1-a_{p}(E) p^{-s}+p^{1-2 s}\right)^{-1} .
$$

This is the $L$-function of an elliptic curve $E$.

Note that the form of the Euler product for $L(E$, s), i.e., the right-hand side of (4), is the same as that of a weight2 modular form. One says that the modular form $f$ of weight 2 is associated to $E$ if $L(f, s)=L(E, s)$; equivalently, if $a_{p}(f)=a_{p}(E)$ for all $p$. That every rational elliptic curve is associated to some modular form of weight 2 was called the Taniyama-Shimura-Weil conjecture, and was proved (for semistable ${ }^{4}$ ) elliptic curves in the famous papers [Wil95] and [TW95] of Wiles and Taylor-Wiles. We direct the reader to Ribet's article [Rib95] for the history of this problem and its connection to Fermat's Last Theorem.

Suppose $X$ is a smooth projective algebraic variety over $\mathbf{Q}$ of dimension $n$ and $0 \leq k \leq 2 n$ is a nonnegative integer. Then one can assign an $L$-function $L\left(H^{k}(X), s\right)$ to the étale cohomology $H_{e t}^{k}\left(X_{\overline{\mathbf{Q}}}, \mathbf{Q}_{\ell}\right)$. For example, if $X=E$ is an elliptic curve and $k=1$, then $L\left(H^{1}(E), s\right)=L(E, s)$ as defined above.

As mentioned above, it is via $L$-functions that number theorists find a bridge between disparate areas of mathematics: algebraic geometry and automorphic forms. For example, here is an important conjecture.

Conjecture 3 (Langlands [Lan80]). Suppose $X$ is a smooth projective variety over $\mathbf{Q}$ and $0 \leq k \leq 2 \operatorname{dim}(X)$. Suppose that the étale cohomology $H_{e t}^{k}\left(X_{\overline{\mathbf{Q}}}, \mathbf{Q}_{\ell}\right)$ affords an irreducible representation of $\operatorname{Gal}(\overline{\mathbf{Q}} / \mathbf{Q})$ of dimension $N$. Then there is an automorphic form $\varphi$ on $\mathrm{GL}_{N}$ so that $L\left(H^{k}(X), s\right)=L(\varphi, s)$.

\section{Exceptional Groups}

Having defined modular forms and automorphic forms, let us now turn our attention to exceptional groups. The Lie groups one meets in practice are often defined as automorphisms of particular algebraic structures. For example, $\mathrm{Sp}(2 n)$ is the automorphism group fixing a symplectic form on a $2 n$-dimensional vector space, and $\mathrm{SO}(p, q)$ is the automorphism group fixing a quadratic form of signature $(p, q)$. These algebraic structures and associated Lie groups come in infinite families, e.g., $\mathrm{Sp}(2 n)$ for $n=1,2,3,4, \ldots$. Because of this fact, they are called classical groups.

It turns out that there are very interesting, exceptional algebraic structures. The exceptional algebraic groups are defined to be automorphism groups of these structures. More precisely, suppose $V$ is a vector space and $\left\{\xi_{\ell}\right\}_{e}$ is

\footnotetext{
${ }^{3}$ It is a theorem of Weil that $a_{p}(E)$ satisfies the bound $\left|a_{p}(E)\right| \leq 2 \sqrt{p}$. In other words, \#E( $\left.\mathbf{F}_{p}\right)$ is approximately $\# \mathbf{P}^{1}\left(\mathbf{F}_{p}\right)$, and $a_{p}(E)$ measures the discrepancy. ${ }^{4}$ The semistability condition was later removed in work of Breuil-ConradDiamond-Taylor.
} 
a finite list of tensors, i.e., $\xi_{\ell} \in \bigotimes_{k} \operatorname{Sym}^{\alpha_{k}^{\ell}}\left(\bigwedge^{k} V\right)$. Associated to this data, one can consider the group $G \subseteq \mathrm{GL}(V)$ that fixes the tensors $\xi_{\ell}$ for all $\ell$. For example, if $V$ is $2 n$ dimensional, then for an appropriate $\omega \in \bigwedge^{2} V, \operatorname{Sp}(2 n)$ is the subgroup of $\mathrm{GL}(V)$ that fixes $\omega$.

If one writes down some random collection of tensors $\xi_{\ell}$ as above, then usually the group $G$ will be trivial, i.e., $G=\{1\}$. However, it sometimes (very rarely) happens that one can write down tensors $\xi_{\ell}$ so that $G$ is nontrivial, and in fact has positive dimension. When these are not the classical groups, one gets the exceptional groups. In this section, we will define these algebraic structures (the $\xi_{\ell}$ ) and their automorphism groups.

5.1. The octonions and $G_{2}$. We begin with the simplest case, that of the octonions and the exceptional group $G_{2}$. The octonions are an 8-dimensional $\mathbf{R}$-vector space $\Theta$, that comes equipped with a multiplication $\Theta \otimes \Theta \rightarrow \Theta$, written as $x, y \mapsto x y$. This multiplication is neither commutative nor associative. However, it does have some redeeming qualities.

First, there is a multiplicative identity $1 \in \Theta$, and $x \cdot 1=$ $1 \cdot x=x$ for all $x \in \Theta$. Most importantly, there is a quadratic norm $n_{\Theta}: \Theta \rightarrow \mathbf{R}$ satisfying $n_{\Theta}(x y)=n_{\Theta}(x) n_{\Theta}(y)$. The group $G_{2}$ is defined as the group fixing this multiplication on $\Theta$ :

$$
G_{2}=\{g \in \mathrm{GL}(\Theta): g(1)=1 \text { and } g(x \cdot y)=g x \cdot g y\} .
$$

To make this more concrete, we give the reader an explicit description of $\Theta$. Namely, one defines $\Theta=M_{2}(\mathbf{R}) \oplus$ $M_{2}(\mathbf{R})$, a direct sum of two copies of the $2 \times 2$-matrices. Suppose $\left(x_{1}, y_{1}\right)$ and $\left(x_{2}, y_{2}\right)$ are in $\Theta$. Then $n_{\Theta}\left(x_{1}, y_{1}\right)=$ $\operatorname{det}\left(x_{1}\right)-\operatorname{det}\left(y_{1}\right)$ is the quadratic norm. The multiplication is defined as

$$
\left(x_{1}, y_{1}\right) \cdot\left(x_{2}, y_{2}\right)=\left(x_{1} x_{2}+y_{2}^{*} y_{1}, y_{2} x_{1}+y_{1} x_{2}^{*}\right) .
$$

Here if $m=\left(\begin{array}{ll}a & b \\ c & d\end{array}\right)$ is a $2 \times 2$ matrix, then $m^{*}=\left(\begin{array}{cc}d & -b \\ -c & a\end{array}\right)$ so that $m+m^{*}=\operatorname{tr}(m) 1_{2}$ and $m m^{*}=\operatorname{det}(m) 1_{2}$. The involution $*$ on $M_{2}(\mathbf{R})$ extends to one on $\Theta$, with similar properties: $(x, y)^{*}=\left(x^{*},-y\right)$. One has $(x, y)+(x, y)^{*}=\operatorname{tr}(x) 1$ and $(x, y)(x, y)^{*}=n_{\Theta}((x, y))$.

The group $G_{2}$ defined by (5) is a noncompact Lie group of dimension 14. There is also a compact form of the group $G_{2}$, which is defined as the automorphisms of an algebra $\Theta^{c}$, closely related to $\Theta$.

To define $\Theta^{c}$, first recall Hamilton's quaternions

$$
\mathbf{H}=\mathbf{R} \oplus \mathbf{R} i \oplus \mathbf{R} j \oplus \mathbf{R} k
$$

with multiplication $i j=k, j k=i, k i=j$. Denote by $*: \mathbf{H} \rightarrow \mathbf{H}$ the involution on $\mathbf{H}$ defined as

$$
(w+x i+y j+z k)^{*}=w-x i-y j-z k,
$$

where $w, x, y, z \in \mathbf{R}$. Now define $\Theta^{c}=\mathbf{H} \oplus \mathbf{H}$ with multiplication

$$
\left(x_{1}, y_{1}\right) \cdot\left(x_{2}, y_{2}\right)=\left(x_{1} x_{2}-y_{2}^{*} y_{1}, y_{2} x_{1}+y_{1} x_{2}^{*}\right) .
$$

The algebra $\Theta^{c}$ is isomorphic to $\Theta$ over $\mathbf{C}$, i.e., $\Theta^{c} \otimes \mathbf{C} \simeq$ $\Theta \otimes \mathbf{C}$. The group $G_{2}^{c}$ is

$$
G_{2}^{c}=\left\{g \in \mathrm{GL}\left(\Theta^{c}\right): g(1)=1 \text { and } g(x \cdot y)=g x \cdot g y\right\} \text {. }
$$

5.2. The groups $F_{4}$ and $E_{6}$. The group $G_{2}$ is the smallest of the exceptional groups. The other exceptional groups are labeled $F_{4}, E_{6}, E_{7}, E_{8}$. They sit in a chain $G_{2} \subseteq F_{4} \subseteq$ $E_{6} \subseteq E_{7} \subseteq E_{8}$, and are of dimensions 14, 52, 78, 133, 248, respectively.

We now define $F_{4}$ and $E_{6}$. To do this, let $H_{3}(\Theta)$ denote the Hermitian $3 \times 3$ matrices with coefficients in the octonions $\Theta$. That is,

$$
H_{3}(\Theta)=\left\{\left(\begin{array}{ccc}
c_{1} & x_{3} & x_{2}^{*} \\
x_{3}^{*} & c_{2} & x_{1} \\
x_{2} & x_{1}^{*} & c_{3}
\end{array}\right): c_{j} \in \mathbf{R}, x_{k} \in \Theta\right\} .
$$

Like the octonions, the 27-dimensional vector space $H_{3}(\Theta)$ has an algebraic structure that has surprisingly many automorphisms. More precisely, $H_{3}(\Theta)$ has a cubic determinant map: if $X \in H_{3}(\Theta)$,

$$
X=\left(\begin{array}{lll}
c_{1} & x_{3} & x_{2}^{*} \\
x_{3}^{*} & c_{2} & x_{1} \\
x_{2} & x_{1}^{*} & c_{3}
\end{array}\right)
$$

set

$$
\begin{aligned}
\operatorname{det}(X)= & c_{1} c_{2} c_{3}-c_{1} n_{\Theta}\left(x_{1}\right)-c_{2} n_{\Theta}\left(x_{2}\right)-c_{3} n_{\Theta}\left(x_{3}\right) \\
& +\operatorname{tr}_{\Theta}\left(\left(x_{1} x_{2}\right) x_{3}\right) .
\end{aligned}
$$

The group $E_{6}$ is defined as the subgroup of $\mathrm{GL}\left(H_{3}(\Theta)\right)$ that fixes this determinant map:

$$
E_{6}=\left\{g \in \mathrm{GL}\left(H_{3}(\Theta)\right): \operatorname{det}(g X)=\operatorname{det}(X) \forall X \in H_{3}(\Theta)\right\} .
$$

The group $F_{4}$ is the subgroup of $E_{6}$ that fixes the element $1_{3}=\operatorname{diag}(1,1,1) \in H_{3}(\Theta)$.

More precisely, there are different "forms" of these exceptional groups. Recall that if $H_{1}$ and $H_{2}$ are real Lie groups, then one says that $H_{2}$ is a "real form" of $H_{1}$ if $\operatorname{Lie}\left(H_{1}\right) \otimes \mathbf{C} \simeq \operatorname{Lie}\left(H_{2}\right) \otimes \mathbf{C}$. Every reductive Lie group has a real form that is compact, and also has a real form that is split. The groups $G_{2}, F_{4}, E_{6}$ that we described above are these split forms. Replacing $\mathrm{H}_{3}(\Theta)$ with $\mathrm{H}_{3}\left(\Theta^{c}\right)$ in the definitions of $F_{4}$ and $E_{6}$ yields different real forms of these groups. By contrast with the case of $G_{2}$, the forms of $F_{4}$ and $E_{6}$ defined in this way are not compact.

5.3. The group $E_{7}$. To define the group $E_{7}$, one proceeds as follows. For ease of notation, set $J=H_{3}(\Theta)$. This notation comes from the fact that $J$ is what is called a "Jordan algebra." Now set $W=\mathbf{R} \oplus J \oplus J^{\vee} \oplus \mathbf{R}$, where $J^{\vee}$ is the linear dual of $J$. This space $W$ is 56-dimensional, and comes equipped with a nondegenerate symplectic form 
and a particular quartic form $Q$ (homogeneous of degree four) which were defined by Freudenthal. The group $E_{7}$ can be defined as the subgroup of $\mathrm{GL}(W)$ that fixes these two forms on $W$.

5.4. The exceptional group $E_{8}$. Every reductive Lie group acts on its Lie algebra by automorphisms. And conversely, if $\mathfrak{g}$ is a (reductive) Lie algebra, then one can define a corresponding Lie group $G$ as

$$
G=\{g \in \mathrm{GL}(\mathfrak{g}):[g X, g Y]=g[X, Y] \forall X, Y \in \mathfrak{g}\} .
$$

All the reductive Lie algebras with the exception of $E_{8}$ have a nontrivial finite-dimensional representation on a vector space smaller than its Lie algebra. For $E_{8}$, its smallest nontrivial representation is the adjoint action on its Lie algebra, so in a sense one must define $E_{8}$ through this action. Fortunately, it is not so difficult to define the Lie algebra $\mathfrak{e}_{8}$.

One can do this as follows [Rum97]. Denote by $V_{3}$ the three-dimensional representation of $\mathfrak{g l}_{3}$. Then

$$
\mathfrak{e}_{8}=\left(\mathfrak{g} l_{3} \oplus \mathfrak{e}_{6}\right) \oplus V_{3} \otimes J \oplus\left(V_{3} \otimes J\right)^{\vee} .
$$

This is a $\mathbf{Z} / 3$-grading with $\mathfrak{g} l_{3} \oplus \mathfrak{e}_{6}$ in degree zero, $V_{3} \otimes J$ in degree one, and $\left(V_{3} \otimes J\right)^{\vee}$ in degree two. The subalgebra $\mathfrak{s} l_{3} \oplus \mathfrak{e}_{6}$ acts on $V_{3} \otimes J$ and $\left(V_{3} \oplus J\right)^{\vee}$ in the obvious manner. For an analogous construction of the Lie algebra $\mathfrak{g}_{2}$, see [FH91, p. 358]. In fact, one can construct all the exceptional Lie algebras using an analogue of (7).

\section{Automorphic Forms on Exceptional Groups}

Having described exceptional groups, and automorphic forms in general, let us now consider automorphic forms on exceptional groups.

6.1. Holomorphic modular forms on $E_{7}$. We first turn our attention to the exceptional group $E_{7}$, as it possesses "modular forms" completely analogous to the classical holomorphic modular forms that we described in the first section. Let $J=H_{3}\left(\Theta^{c}\right)$, and let $G$ denote the exceptional group of type $E_{7}$ defined as in subsection 5.3. The group $G$ is neither split nor compact. For this group, if $K_{G}$ denotes the maximal compact of $G(\mathbf{R})$, the symmetric space $G(\mathbf{R}) / K_{G}$ has a $G(\mathbf{R})$-invariant complex structure.

Denote by $J_{+}$the subset of $J$ consisting of elements of the form $Y^{2}$ for some $Y \in J$ with $\operatorname{det}(Y) \neq 0$. (If $Y \in J$, then its square $Y^{2}$-in the sense of usual matrix multiplication-is also an element of $J$.) The symmetric space $G(\mathbf{R}) / K_{G}$ can be identified with

$$
\mathfrak{h}_{J}=\left\{Z=X+i Y: X \in J, Y \in J_{+}\right\} .
$$

Consequently, there is an action of $G(\mathbf{R})$ on $\mathfrak{h}_{J}$, which one should think of as generalized (exceptional) linear fractional transformations.

As the reader will no doubt have noticed, the space $\mathfrak{h}_{J}$ is very similar to the complex upper half-space $\mathfrak{h}$. And indeed, the group $G$ possesses special automorphic forms- the modular forms-that are close cousins of the classical modular forms on $\mathrm{SL}_{2}$ defined in section 2. Just as in the classical case, there is a function $j: G(\mathbf{R}) \times \mathfrak{h}_{J} \rightarrow \mathfrak{h}_{J}$ satisfying $j\left(g_{1} g_{2}, Z\right)=j\left(g_{1}, g_{2} Z\right) j\left(g_{2}, Z\right)$ for all $g_{1}, g_{2} \in G(\mathbf{R})$. To define the modular forms, suppose $\Gamma$ is an arithmetic subgroup of $G(\mathbf{R})$ and $k \geq 0$ is an integer. A modular form of weight $k$ for $\Gamma$ is a holomorphic function $f: \mathfrak{h}_{J} \rightarrow \mathbf{C}$ on $\mathfrak{h}_{J}$ satisfying $f(\gamma Z)=j(\gamma, Z)^{k} f(Z)$ for all $\gamma \in \Gamma$ and for which the $\Gamma$-invariant function

$$
\left|\operatorname{det}(Y)^{k / 2} f(Z)\right|=\left|j\left(g, i 1_{3}\right)^{-k} f\left(g \cdot i 1_{3}\right)\right|
$$

is of moderate growth on $G(\mathbf{R})$.

These modular forms have a "q-expansion," completely analogous to the Fourier expansion of classical modular forms. Assume for simplicity that $\Gamma=G(\mathbf{Z})$ is the maximal arithmetic subgroup of $G(\mathbf{R})$. Fix a certain lattice $J_{\mathbf{Z}}$ in $J$, and let $J_{\mathbf{Z}}^{+}$denote the intersection of $J_{\mathbf{Z}}$ with the closure of $J_{+}$in $J_{\mathbf{R}}$. If $f$ is a modular form for $\Gamma$, then

$$
f(Z)=\sum_{T \in J_{\mathbf{Z}}^{+}} a_{f}(T) e^{2 \pi i \operatorname{tr}(T Z)}
$$

for complex numbers $a_{f}(T)$; this is its Fourier expansion.

Some examples of modular forms on $G$ can be found in work of Baily [Bai70], Kim [Kim93], and Kim-Yamauchi [KY16]. However, let us reiterate that very little is known about them. For example, the $L$-functions $L(f, s)$ of modular forms on $G$ are known to have meromorphic continuation in $s$ [Lan71]. However, the conjectured finer analytic properties of these $L$-functions, such as the finiteness of its poles, are not known.

6.2. Quaternionic modular forms. Of the exceptional Dynkin types, $E_{7}$ is special in that the types $G_{2}, F_{4}, E_{6}$, and $E_{8}$ do not have real forms $G$ so that the associated symmetric space $X_{G}$ has "modular forms" similar to the classical holomorphic modular forms. ${ }^{5}$ It turns out there is a certain type of nonholomorphic "quaternionic" modular forms on one of the real forms of each of the exceptional Dynkin types

$$
G_{2} \subseteq F_{4} \subseteq E_{6} \subseteq E_{7} \subseteq E_{8} .
$$

These were defined by Gross and Wallach [GW96] and Gan-Gross-Savin [GGS02], and have subsequently been studied by Gan, Loke, Weissman, and the author. Among their nice features are that they have a Fourier expansion with some of the same good properties of classical modular forms [Pol20], and that they behave well under pullback in the sequence (9).

Instead of being $\mathbf{C}$-valued functions on an exceptional group $G$, quaternionic modular forms are naturally valued in the finite-dimensional representations of the compact Lie group $\mathrm{SU}(2) /\{ \pm 1\}$. More precisely, if $n \geq 1$ is

\footnotetext{
${ }^{5}$ There is a real form of $E_{6}$ whose symmetric space has hermitian structure, but the automorphic forms on this $E_{6}$ do not have Fourier expansions similar to that of (8).
} 
an integer, a quaternionic modular form of weight $n$ on a quaternionic exceptional group $G$ is a function $f: \Gamma \backslash G \rightarrow$ $\operatorname{Sym}^{2 n}\left(\mathbf{C}^{2}\right)$ satisfying conditions analogous to those of Definition 1 , together with being annihilated by a specific firstorder linear differential operator $\mathcal{D}_{n}$, i.e., $\mathcal{D}_{n} f=0$. Here $\operatorname{Sym}^{2 n}\left(\mathbf{C}^{2}\right)$ is the $(2 n+1)$-dimensional irreducible representation of SU(2). Similar to how the Cauchy-Riemann solutions force the nice form of the Fourier expansion of holomorphic modular forms, being annihilated by $\mathcal{D}_{n}$ forces the quaternionic modular form $f$ to have a robust Fourier expansion. The robustness of the Fourier expansion allows one to ask (and answer ${ }^{6}$ ) questions such as "Do there exist quaternionic modular forms on $E_{8}$ and $G_{2}$ whose Fourier coefficients are all integers?"

One beautiful example of such a quaternionic modular form is a function $\theta_{\text {min }}$ on $E_{8}$ of weight 4 , defined and studied by Gan [Gan00]. The function $\theta_{\min }$ can be thought of as an $E_{8}$-analogue of the classical theta function $\theta(z)=\sum_{n \in \mathbf{Z}} q^{n^{2}}$.

Another source of examples of these quaternionic modular forms comes from Eisenstein series associated to holomorphic cusp forms. Precisely, if $n \geq 6$ and $\varphi$ is a classical holomorphic modular cusp form of weight $3 n$, then one can construct out of $\varphi$ an Eisenstein series $E(\varphi)$ on $G_{2}$ that gives a modular form of weight $n$. The fact that $\varphi$ is holomorphic is crucial to $E(\varphi)$ being annihilated by $\mathcal{D}_{n}$.

6.3. Automorphic forms on split exceptional groups. Our treatment of automorphic forms in this article has been biased towards those that are most similar to the classical holomorphic modular forms that one first meets, in terms of having a robust Fourier expansion. This bias is a bit out-of-line with the development of the theory of automorphic forms. In this final subsection, we go back to the mainstream and highlight some work on automorphic forms on split exceptional groups, for which there is not a similarly robust theory of the Fourier expansion. In no way should our selection of topics here be considered exhaustive.

6.3.1. L-functions. Relatively speaking, much more is known about the $L$-functions of automorphic forms on split exceptional groups than on their nonsplit counterparts. To explain the state of affairs in just a little more detail, recall that split reductive algebraic groups $G$ possess generic cusp forms. Roughly speaking, a generic cusp form is a cuspidal automorphic form which possesses nontrivial averages over large unipotent groups $U^{\prime}$. That is, if $\varphi$ is cuspidal and generic, then the integral $\int_{\left(U^{\prime} \cap \Gamma\right) \backslash U^{\prime}} \varphi(u g) d u$ is not identically 0 as a function of $g \in G$. Here, for the more knowledgeable reader, we point out that $U^{\prime}=[U, U]$ is the commutator subgroup of a maximal unipotent group $U$.

\footnotetext{
${ }^{6}$ The answer is "Yes."
}

Generic cuspidal automorphic forms are very far from being holomorphic: many one-dimensional unipotent averages of $\varphi$ vanish identically if $\varphi$ is a holomorphic cuspidal modular form. Similarly, certain three-dimensional unipotent averages of $\varphi$ vanish identically if $\varphi$ is a quaternionic cuspidal modular form, in the sense of subsection 6.2 .

The $L$-functions of generic cuspidal automorphic forms on the split exceptional groups $G_{2}, F_{4}, E_{6}$, and $E_{7}$ have been studied by Ginzburg, Ginzburg-Rallis, and PiatetskiShapiro-Rallis-Schiffmann. For example, in [Gin95] it is proved that the so-called standard $L$-function $L(\pi, S t d, s)$ of cuspidal generic automorphic representations on split $E_{7}$ has meromorphic analytic continuation with at most two simple poles.

6.3.2. Theta functions. As a rule of thumb, constructing automorphic forms is difficult. The first explicit examples one tends to write down are often constructed using $\theta$ functions and their generalizations. For example, taking the classical $\theta$ function $\theta(z)=\sum_{n \in \mathbf{Z}} q^{n^{2}}$ and considering $\theta(z)^{4}$ gives the modular form $\theta_{4}(z)$ of section 1 .

The function $\theta(z)$ has the property that many of its Fourier coefficients are 0 : the coefficient of $q^{N}$ is nonzero only when $N$ is a square. Many reductive groups, and in particular the split exceptional groups $F_{4}, E_{6}, E_{7}$, and $E_{8}$, possess analogues of this function $\theta(z)$. Such an analogue goes by the moniker of a minimal automorphic form, and possesses the property that very many of its generalized Fourier coefficients are 0 . We direct the reader to work of Ginzburg-Rallis-Soudry [GRS97] and Ginzburg for results on these minimal automorphic forms.

Besides $\theta$ functions themselves, one can use theta functions as integral kernel functions to create other automorphic forms. Without going into the details of this procedure, let us simply mention the very interesting examples of Rallis-Schiffmann, Li-Schwermer, and Gan-GurevichJiang, which use such a construction to create special automorphic forms on the split exceptional group $G_{2}$.

6.3.3. Further directions. Finally, we mention that there has been some activity to relate the Fourier coefficients of automorphic functions on split exceptional groups to quantities that arise in string theory. We direct the interested reader to the book [FGKP18] and the numerous references contained therein.

\section{A. Technical Details on Exceptional Groups}

In this appendix we include a few technical details on exceptional groups that might be useful to the interested reader.

A.1. Real forms of exceptional groups. In section 5 we defined various forms of the exceptional groups. We remark that the forms of $G_{2}, F_{4}, E_{6}, E_{7}, E_{8}$ that are defined there are the simply connected algebraic groups. (The 
algebraic groups $G_{2}, F_{4}$, and $E_{8}$ are both adjoint and simply connected, while the simply connected $E_{6}$ and $E_{7}$ have centers the cyclic groups of orders 3 and 2, respectively.) We connect what was written above to other standard notation for these groups.

- The real form of $F_{4}$ defined in section 5 using $H_{3}(\Theta)$ is the split form $F_{4(4)}$ if $\Theta$ is the split octonions and is the compact form $F_{4(-52)}$ if defined using $\mathrm{H}_{3}\left(\Theta^{c}\right)$.

- The real forms of $E_{6}$ defined in section 5 are the split form if defined using $H_{3}(\Theta)$ and the form $E_{6(-26)}$ if defined using $H_{3}\left(\Theta^{c}\right)$.

- The real forms of $E_{7}$ defined in section 5 are the split form if defined using $J=H_{3}(\Theta)$ and the form $E_{7(-25)}$ if defined using $J=H_{3}\left(\Theta^{c}\right)$.

- The real forms of $E_{8}$ defined in section 5 are the split form if defined using $J=H_{3}(\Theta)$ and the form $E_{8(-24)}$ if defined using $J=H_{3}\left(\Theta^{c}\right)$.

In section 5 it was mentioned that every exceptional Lie algebra can be constructed in a form analogous to (7). To do this, one lets the Jordan algebra (or precisely, cubic norm structure) $J$ vary, and the Lie algebra $\mathfrak{e}_{6}$ is replaced with the Lie algebra $\mathfrak{m}(J)$ of the group of determinantpreserving linear maps on $J$. One obtains a Lie algebra

$$
\mathfrak{g}(J)=\left(\mathfrak{g l}_{3} \oplus \mathfrak{m}(J)\right) \oplus V_{3} \otimes J \oplus\left(V_{3} \otimes J\right)^{\vee},
$$

where now $J$ is an arbitrary cubic norm structure, instead of just the exceptional cubic norm structure $H_{3}\left(\Theta^{c}\right)$. If $J$ varies over cubic norm structures with positive-definite trace forms, one constructs (uniformly) the so-called quaternionic forms of the exceptional groups. Details can be found in, for example, [Pol20]. With $J=H_{3}(\mathbf{H}), \mathfrak{g}(J)$ is the quaternionic $\mathfrak{e}_{7}$ (also known as $\mathfrak{e}_{7(-5)}$ ); if $J=H_{3}(\mathbf{C})$, then $\mathfrak{g}(J)$ is the quasisplit and quaternionic $\mathfrak{e}_{6}$ (also known as $\left.\mathfrak{e}_{6(2)}\right)$; and if $J=H_{3}(\mathbf{R})$, then $\mathfrak{g}(J)$ is the split $\mathfrak{f}_{4}$.

A.2. The definition of $E_{7}$. It is not difficult to be even more precise about the definition of the exceptional groups $E_{7}$ and $E_{8}$ given above. To do so, we require one additional piece of notation for the Jordan algebra $J=H_{3}(\Theta)$; namely, a symmetric bilinear map $\times: J \otimes J \rightarrow J^{\vee}$ defined as follows. Polarizing the determinant map det : $J \rightarrow \mathbf{R}$, there is a symmetric trilinear map $(\cdot, \cdot, \cdot)$ on $J$ satisfying $(X, X, X)=6 \operatorname{det}(X)$. Now, for $X, Y$ in $J$, define $X \times Y \in J^{\vee}$ (the linear dual of $J$ ) via $(X \times Y)(Z)=(X, Y, Z)$. Set $X^{\#}=\frac{1}{2} X \times X$. There is an identically defined map $\times: J^{\vee} \otimes J^{\vee} \rightarrow J$.

With this bit of notation, we can now precisely define the symplectic and quartic forms on $W_{J}$ fixed by $E_{7}$. For $E_{7}$, suppose $(a, b, c, d) \in W$, so that $a, d \in \mathbf{R}, b \in J$, and $c \in J^{\vee}$, and similarly for $\left(a^{\prime}, b^{\prime}, c^{\prime}, d^{\prime}\right)$. Then the symplectic form $\langle\cdot, \cdot\rangle$ on $W$ is given by $\left\langle(a, b, c, d),\left(a^{\prime}, b^{\prime}, c^{\prime}, d^{\prime}\right)\right\rangle=a d^{\prime}-$ $\left(b, c^{\prime}\right)+\left(b^{\prime}, c\right)-d a^{\prime}$ and the quartic form $Q$ on $W$ is given by

$$
\begin{aligned}
Q((a, b, c, d))= & (a d-(b, c))^{2}+4 a \operatorname{det}(c) \\
& +4 d \operatorname{det}(b)-4\left(c^{\#}, b^{\#}\right) .
\end{aligned}
$$

A.3. The definition of $E_{8}$. It is also possible to concisely define the Lie bracket on $\mathfrak{e}_{8}$. Because $V_{3}$ is a representation of $\xi_{3}$, there are identifications $\bigwedge^{2} V_{3} \simeq V_{3}^{\vee}$ and $\bigwedge^{2} V_{3}^{\vee} \simeq$ $V_{3}$. Now if $u, u^{\prime} \in V_{3}$ and $X, X^{\prime} \in J$, then $\left[u \otimes X, u^{\prime} \otimes X^{\prime}\right]=$ $\left(u \wedge u^{\prime}\right) \otimes\left(X \times X^{\prime}\right)$, considered as an element of $\left(V_{3} \otimes J\right)^{\vee}$. The Lie bracket $[\cdot, \cdot]:\left(V_{3} \otimes J\right)^{\vee} \otimes\left(V_{3} \otimes J\right)^{\vee} \rightarrow V_{3} \otimes J$ is defined analogously. Finally, the map $\left(V_{3} \otimes J\right) \otimes\left(V_{3} \otimes J\right)^{\vee}$ induced by the Lie bracket is defined using the maps $V_{3} \otimes V_{3}^{\vee} \simeq$ $\operatorname{End}\left(V_{3}\right) \rightarrow \mathfrak{s} l_{3}$ and a bilinear map $\Phi^{0}: J^{\vee} \otimes J \rightarrow \mathfrak{e}_{6}$ defined as

$$
\Phi_{c, b}^{0}(X)=-c \times(b \times X)+(c, X) b+\frac{1}{3}(b, c) X .
$$

Here $c \in J^{\vee}$ and $b, X \in J$.

ACKNOWLEDGMENT. It is a pleasure to thank the referees for their careful reading of this manuscript and thoughtful suggestions.

\section{References}

[Bai70] Walter L. Baily Jr., An exceptional arithmetic group and its Eisenstein series, Ann. of Math. (2) 91 (1970), 512-549, DOI 10.2307/1970636, MR269779

[Dir37] P. G. L. Dirichlet, Beweis des satzes, dass jede unbegrenzte arithmetische progression, deren erstes glied und differenz ganze zahlen ohne gemeinschaftlichen factor sind, unendlich viele primzahlen enthält, Abhandlungen der Königlichen Preußischen Akademie der Wissenschaften zu Berlin 48 (1837), 45-71.

[FGKP18] Philipp Fleig, Henrik P. A. Gustafsson, Axel Kleinschmidt, and Daniel Persson, Eisenstein series and automorphic representations: With applications in string theory, Cambridge Studies in Advanced Mathematics, vol. 176, Cambridge University Press, Cambridge, 2018. MR3793195

[FH91] William Fulton and Joe Harris, Representation theory: A first course, Graduate Texts in Mathematics, vol. 129, Springer-Verlag, New York, 1991. Readings in Mathematics. MR1153249

[Gan00] Wee Teck Gan, A Siegel-Weil formula for exceptional groups, J. Reine Angew. Math. 528 (2000), 149-181, DOI 10.1515/crll.2000.088 MR1801660

[GGS02] Wee Teck Gan, Benedict Gross, and Gordan Savin, Fourier coefficients of modular forms on $G_{2}$, Duke Math. J. 115 (2002), no. 1, 105-169, DOI 10.1215/S0012-7094-0211514-2. MR1932327

[Gin95] David Ginzburg, On standard L-functions for $E_{6}$ and $E_{7}$, J. Reine Angew. Math. 465 (1995), 101-131, DOI 10.1515/crll.1995.465.101 MR1344132

[GRS97] David Ginzburg, Stephen Rallis, and David Soudry, On the automorphic theta representation for simply laced groups, Israel J. Math. 100 (1997), 61-116, DOI 10.1007/BF02773635. MR1469105 
[GW96] Benedict H. Gross and Nolan R. Wallach, On quaternionic discrete series representations, and their continuations, J. Reine Angew. Math. 481 (1996), 73-123, DOI 10.1515/crll.1996.481.73 MR1421947

[Kim93] Henry H. Kim, Exceptional modular form of weight 4 on an exceptional domain contained in $\mathbf{C}^{27}$, Rev. Mat. Iberoamericana 9 (1993), no. 1, 139-200, DOI 10.4171/RMI/134. MR1216126

[KY16] Henry H. Kim and Takuya Yamauchi, Cusp forms on the exceptional group of type $E_{7}$, Compos. Math. 152 (2016), no. 2, 223-254, DOI 10.1112/S0010437X15007538 MR3462552

[Lan80] R. P. Langlands, L-functions and automorphic representations, Proceedings of the International Congress of Mathematicians (Helsinki, 1978), Acad. Sci. Fennica, Helsinki, 1980, pp. 165-175. MR562605

[Lan71] Robert P. Langlands, Euler products, Yale University Press, New Haven, Conn.-London, 1971. A James K. Whittemore Lecture in Mathematics given at Yale University, 1967; Yale Mathematical Monographs, 1. MR0419366

[Pol20] Aaron Pollack, The Fourier expansion of modular forms on quaternionic exceptional groups, Duke Math. J. 169 (2020), no. 7, 1209-1280, DOI 10.1215/U0127094-2019-0003 MR4094735

[Rib95] Kenneth A. Ribet, Galois representations and modular forms, Bull. Amer. Math. Soc. (N.S.) 32 (1995), no. 4, 375-402, DOI 10.1090/S0273-0979-1995-00616-6 MR1322785

[Rum97] Karl E. Rumelhart, Minimal representations of exceptional p-adic groups, Represent. Theory 1 (1997), 133-181, DOI 10.1090/S1088-4165-97-00009-5. MR14551 28

[Ser73] J.-P. Serre, A course in arithmetic, Graduate Texts in Mathematics, vol. 7, Springer-Verlag, New York-Heidelberg, 1973. Translated from the French. MR0344216

[TW95] Richard Taylor and Andrew Wiles, Ring-theoretic properties of certain Hecke algebras, Ann. of Math. (2) 141 (1995), no. 3, 553-572, DOI 10.2307/2118560 MR1333036

[Wil95] Andrew Wiles, Modular elliptic curves and Fermat's last theorem, Ann. of Math. (2) 141 (1995), no. 3, 443-551, DOI 10.2307/2118559. MR1333035

[Zag08] Don Zagier, Elliptlc modular forms and their applications, The 1-2-3 of modular forms, Universitext, Springer, Berlin, 2008, pp. 1-103, DOI 10.1007/978-3-540-74119. 0_1 MR2409678

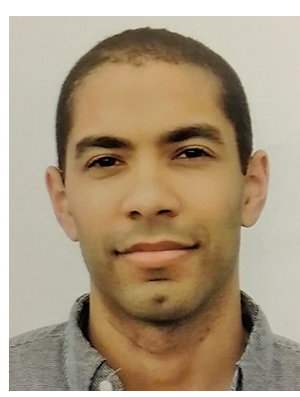

Aaron Pollack

Credits

The opening image is courtesy of brightstars via Getty. Photo of the author is courtesy of the author.

When wers the lass time pou wisited the AMS Bookstore?

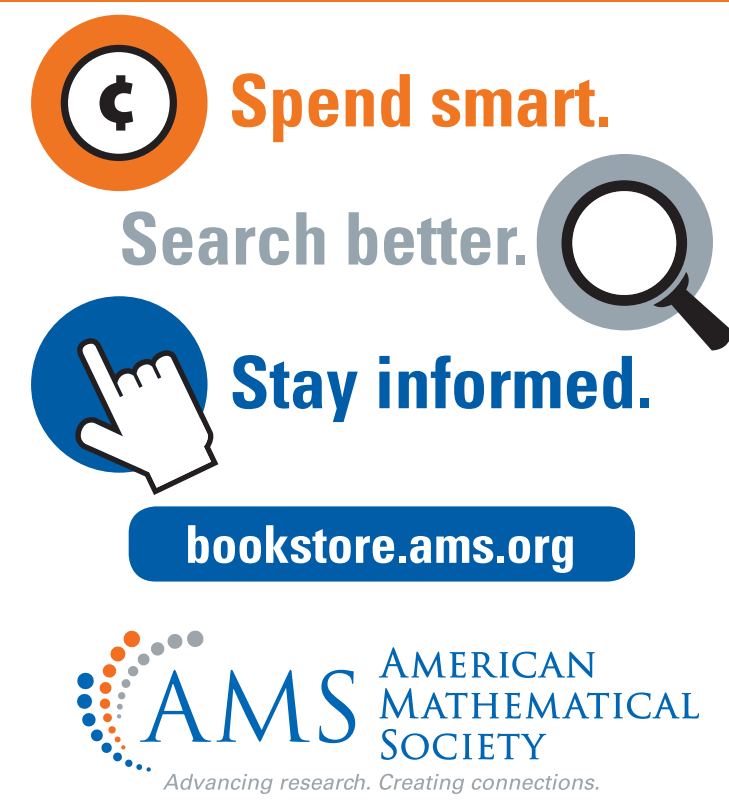

